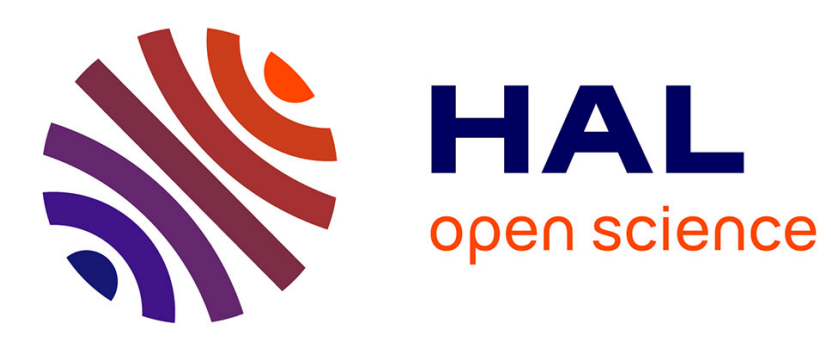

\title{
Sur l'efficacité des ions et des électrons dans un plasma froid venant en contact avec un polymère
}

\author{
P. Gomes de Lima, J. Lopez, Bernard Despax, C. Mayoux
}

\section{To cite this version:}

P. Gomes de Lima, J. Lopez, Bernard Despax, C. Mayoux. Sur l'efficacité des ions et des électrons dans un plasma froid venant en contact avec un polymère. Revue de Physique Appliquée, 1989, 24

(3), pp.331-335. 10.1051/rphysap:01989002403033100 . jpa-00246054

\section{HAL Id: jpa-00246054 \\ https://hal.science/jpa-00246054}

Submitted on 1 Jan 1989

HAL is a multi-disciplinary open access archive for the deposit and dissemination of scientific research documents, whether they are published or not. The documents may come from teaching and research institutions in France or abroad, or from public or private research centers.
L'archive ouverte pluridisciplinaire HAL, est destinée au dépôt et à la diffusion de documents scientifiques de niveau recherche, publiés ou non, émanant des établissements d'enseignement et de recherche français ou étrangers, des laboratoires publics ou privés. 
Classification

Physics Abstracts

$52.40-81.40$

\title{
Sur l'efficacité des ions et des électrons dans un plasma froid venant en contact avec un polymère
}

\author{
P. Gomès de Lima, J. Lopez, B. Despax et C. Mayoux \\ Laboratoire de Génie Electrique, Associé au C.N.R.S. (UA 304), Université Paul Sabatier, 118 route de \\ Narbonne, 31062 Toulouse Cedex, France
}

(Reçu le $1^{\text {er }}$ décembre 1987, révisé le 8 novembre 1988, accepté le 8 décembre 1988)

\begin{abstract}
Résumé. - Un dispositif expérimental mis au point récemment permet d'irradier une cible par les différentes espèces rencontrées dans un plasma froid. D'autre part, des mesures électriques pratiquées in situ soit sous vide de $10^{-4} \mathrm{~Pa}$ soit à la pression atmosphérique en atmosphère inerte caractérisent les conséquences de l'impact des ions et des électrons de faible énergie (50-300 eV) agissant séparément ou en conjugaison. Plusieurs matériaux sont actuellement étudiés (polyimide, polyepoxy, polyoléfines). Seuls les résultats concernant le polypropylène seront présentés ici. Des analyses complémentaires pratiquées ex situ, telles que la RPE ou la mesure de courants de fuite confirment l'efficacité des ions ainsi que celle de l'association des ions et des électrons.
\end{abstract}

\begin{abstract}
An experimental set up recently achieved allows a target irradiation by the different species existing in a cold plasma. On the other hand electrical measurements in situ, under $10^{-4} \mathrm{~Pa}$ vacuum or atmospheric pressure in inert gas, characterize the effect of low energy ions and electrons (50-300 eV) working separately or in conjunction. Actually several materials are studied (polyimide, epoxy, resins, polyolefins), results on polypropylene are only presented. Complementary analysis carried out ex situ, such as ESR or leakage currents measurement confirm the ions efficiency and the combination of ions and electrons as well.
\end{abstract}

\section{Introduction.}

Le comportement des polymères et plus particulièrement de ceux utilisés dans le domaine de l'isolation électrique lorsqu'ils sont soumis à des irradiations de faible énergie (50-300 eV) a déjà fait l'objet d'un certain nombre d'études dans le passé [1]. Historiquement c'est aux processus de dégradation des matériaux isolants qu'il s'est avéré utile de s'intéresser. Il est certain que les méfaits causés par des défauts, se présentant sous forme de bulles de gaz, dans les câbles, les alternateurs, les condensateurs, les transformateurs ou plus récemment dans les systèmes embarqués sur fusée ou satellite, ont préoccupé les utilisateurs dès le début de ce siècle. C'est ainsi qu'en 1936 D. M. Robinson [2] dont l'intérêt s'était porté sur les câbles haute tension imprégnés d'huile mettait déjà en cause la présence de cavités gazeuses et le bombardement de leurs parois par des ions. Par la suite, les travaux se sont multipliés sur ce thème.

Cependant, l'interaction d'un plasma froid avec un matériau diélectrique n'est pas nécessairement nocive lorsqu'elle est de courte durée. D'une manière générale elle améliore l'adhérence entre matériaux et ce fait est bien connu.

Cet aspect positif d'un plasma froid agissant sur un matériau diélectrique est par exemple utilisé dans le domaine de la télécopie qui utilise un procédé plus direct que le procédé «xerox » avec la formation d'une image électrostatique directement sur une feuille de papier. La réussite de ce procédé nécessite la résolution complète de plusieurs problèmes dont en particulier : l'optimisation des charges déposées par effet couronne, l'amélioration du temps pendant lequel les charges demeurent sur la surface du papier, ce phénomène étant lié à la conduction en volume du matériau. Les charges déposées sous forme ponctuelle sont matérialisées par un révélateur et la juxtaposition de plusieurs points crée une lettre. Il s'agit donc d'une électrophotographie.

Un deuxième exemple peut être cité, il concerne le traitement de films de polymères sur lesquels on souhaite écrire ou déposer une couche métallique ; dans ce dernier cas la feuille métallique si elle est bobinée autour d'un mandrin constituera un condensateur. 
Qu'il s'agisse de la dégradation des matériaux isolants ou de leur transformation en surface une meilleure compréhension de l'action du plasma peut contribuer à une amélioration des matériaux ou des procédés de mise en œuvre ainsi qu'à une utilisation plus rationnelle des traitements de surface.

L'étude que nous avons entreprise consiste à observer les modifications que subissent les matériaux lorsqu'ils sont irradiés tout d'abord séparément par les différentes espèces rencontrées dans un plasma puis lorsqu'ils subissent l'action de ces mêmes espèces envoyées simultanément et en multipliant les combinaisons. S'il est sûr que chaque espèce a une action spécifique on souhaite aussi montrer que l'une d'entre elles a un rôle catalytique.

Nous présentons ici les observations faites sur un polypropène biétiré à plat de $8 \mu \mathrm{m}$ d'épaisseur, chacun des échantillons ayant été irradié par des ions et des électrons de faible énergie dans l'enceinte que nous avons convenu d'appeler IRMA (IRradiation de MAtériaux).

\section{Dispositif expérimental.}

Il comprend, pour le présent travail, une source d'ions, une source d'électrons et un système de deux électrodes permettant de réaliser des décharges électriques dans les mêmes conditions de température et de pression que les irradiations du matériau. D'autre part, la caractérisation électrique peut être faite grâce à une sonde électrostatique donnant accès aux charges de surface et à leur durée de vie, puis à l'aide d'une électrode mobile qui permet d'effectuer les mesures de pertes diélectriques et de conductivité.

Les systèmes d'irradiation et de mesure sont placés sur un support en aluminium et peuvent se déplacer suivant l'axe des $\mathrm{X}$ ainsi que le montre la figure 1. En face de ce support, se présente un disque sur lequel sont fixées quatre électrodes en laiton, dont une peut être chauffée jusqu'à la température de $200^{\circ} \mathrm{C}$. Le disque monté sur une traversée tournante permet de faire succéder une mesure électrique à une irradiation et réciproquement.

Cet ensemble est placé dans une enceinte à l'intérieur de laquelle on réalise un vide de $10^{-4} \mathrm{~Pa}$ lorsque les sources ne fonctionnent pas et de $5 \times 10^{-4} \mathrm{~Pa}$ au moment de l'irradiation. Les densités de courant fournies par les sources d'ions et d'électrons sont $0,5 \mu \mathrm{A} / \mathrm{mm}^{2}$ et $0,25 \mu \mathrm{A} / \mathrm{mm}^{2}$ respectivement.

\section{Résultats expérimentaux.}

3.1 Pertes diéleCtriques. - L'évolution de cette caractéristique de l'isolant a été étudiée en fonction du temps d'irradiation, de l'énergie des espèces incidentes et de l'atmosphère dans l'enceinte de bombardement.

La figure 2 permet de constater que tg $\delta$ dépend du temps d'irradiation du film de polypropylène considéré et que sa valeur est plus élevée dans la région de la fréquence la plus basse c'est-à-dire

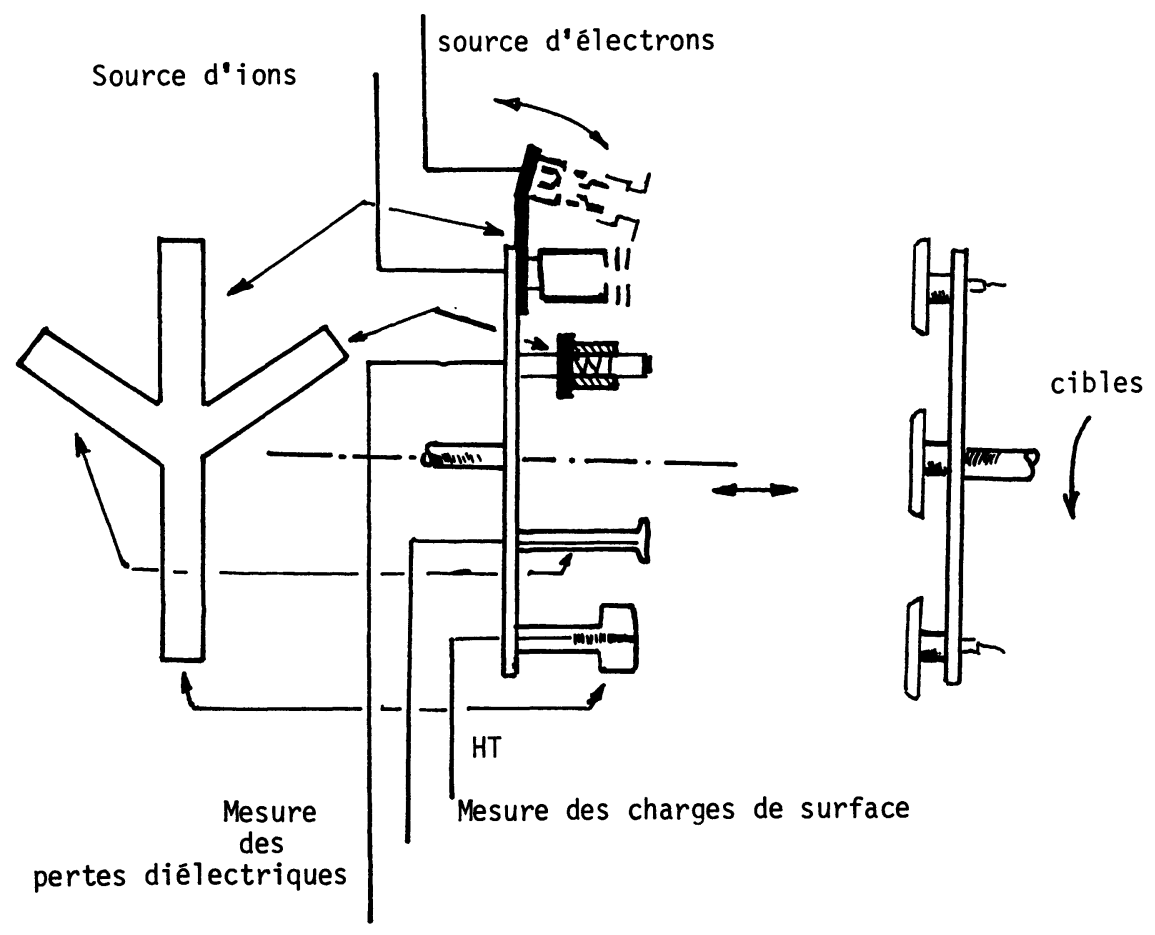

Fig. 1. - Experimental set up. 


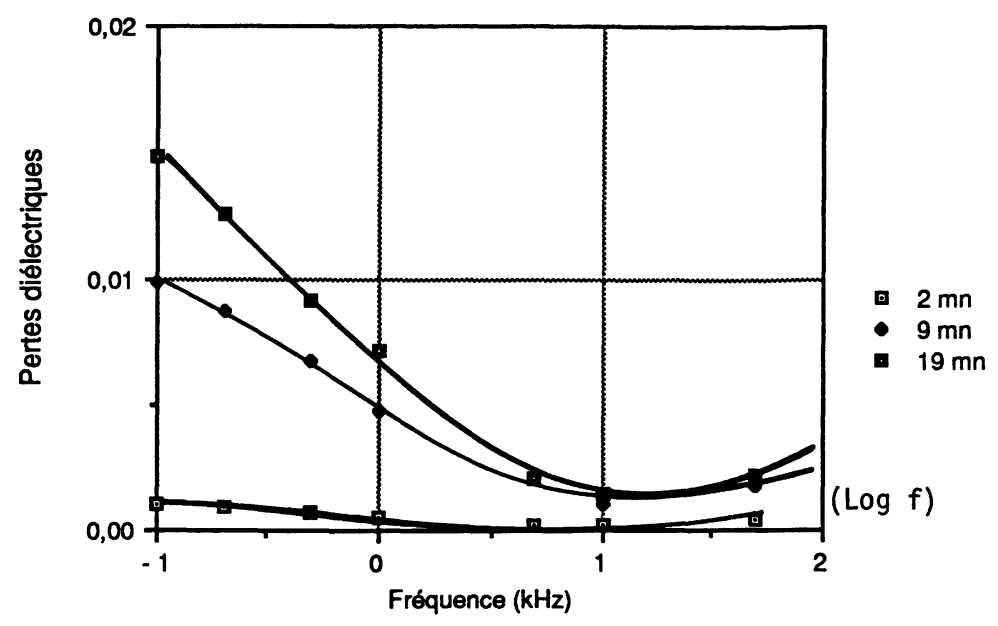

Fig. 2. - Evolution of polypropylene dielectric losses versus frequency, for several times of irradiation.

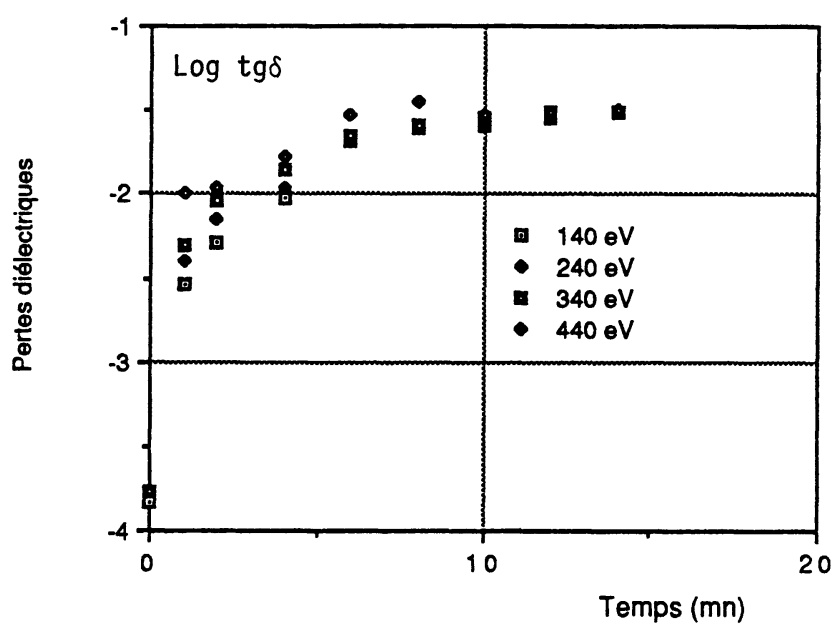

Fig. 3. - Evolution of dielectric losses, at $5 \mathrm{kHz}$, versus irradiation time.

$100 \mathrm{~Hz}$. Lorsqu'on souhaite suivre l'évolution des pertes diélectriques en fonction d'un certain nombre de paramètres on choisit une fréquence de mesure le plus souvent $1 \mathrm{kHz}$. Il apparaît alors deux cinétiques de transformation: entre 0 et 4 min d'irradiation l'évolution des pertes est très importante et dépend de l'énergie des ions, au-delà de ce temps les pertes semblent se stabiliser et l'énergie des espèces joue un rôle moins important.

Il a été noté au cours des expériences que tg $\delta$, pour une énergie donnée, prend une valeur sensiblement différente selon que l'irradiation de la cible a été effectuée d'une manière continue, durant $10 \mathrm{~min}$, par exemple, ou d'une manière séquencée c'est-à-dire cinq fois 2 min. On peut imaginer deux causes à l'origine de ce phénomène : soit la décharge partielle de l'échantillon entre deux irradiations et ce par l'intermédiaire de l'électrode de mesure, soit l'élévation de température du polymère lorsqu'il est irradié sans interruption. En effet, nous avons constaté une élévation de température d'environ $12^{\circ} \mathrm{C}$ en plaçant une résistance de platine au contact de l'échantillon.

L'introduction dans la source d'ions, de gaz tels que l'argon, l'azote ou l'oxygène ont permis de comparer les effets de particules de taille différente, mais accélérées sous la même tension. Il est apparu [3] que les pertes diélectriques provoquées par les ions argon étaient plus importantes que celles générées par les ions azote. Nous avons par ailleurs remarqué que les électrons, que l'on peut considérer comme des particules de masse infiniment plus faible, ne provoquaient pas de variations significatives de la valeur de $\operatorname{tg} \delta$.

Enfin, nous avons noté l'influence de l'atmosphère résiduelle dans l'enceinte d'irradiation après que celle-ci soit achevée. Nous avons en particulier observé que la présence d'un gaz inerte tel que l'argon, à une pression d'environ $10^{-1} \mathrm{~Pa}$ ne modifie pas la valeur des pertes diélectriques. Au contraire lorsque le gaz est de l'oxygène ou de l'azote une variation de cette même caractéristique électrique est observée.

3.2 RADICAUX LIBRES. - La détection de la présence éventuelle de radicaux libres dans le polypropylène qui a subi une irradiation par les espèces que nous étudions, ne peut se faire qu'à l'extérieur de l'enceinte de bombardement. Après son irradiation l'échantillon de polymère est introduit dans le tube de quartz destiné à être placé dans la cavité résonante. L'introduction, le scellement et la mise dans l'azote liquide nécessitent $10 \mathrm{~min}$ environ. La raie $\mathrm{Fe}^{3+}$ dans un monocristal de $\mathrm{MgO}$ placé dans la cavité résonante a été utilisée comme référence. Ce sont donc des mesures relatives qui ont été réalisées à $100 \mathrm{~K}$ en comparant la hauteur Ipp du signal correspondant aux radicaux, à la raie de références $\mathrm{Fe}^{3+}$. La figure 4 montre les spectres relatifs à des irradiations d'ions obtenus à partir de l'oxygène, de 


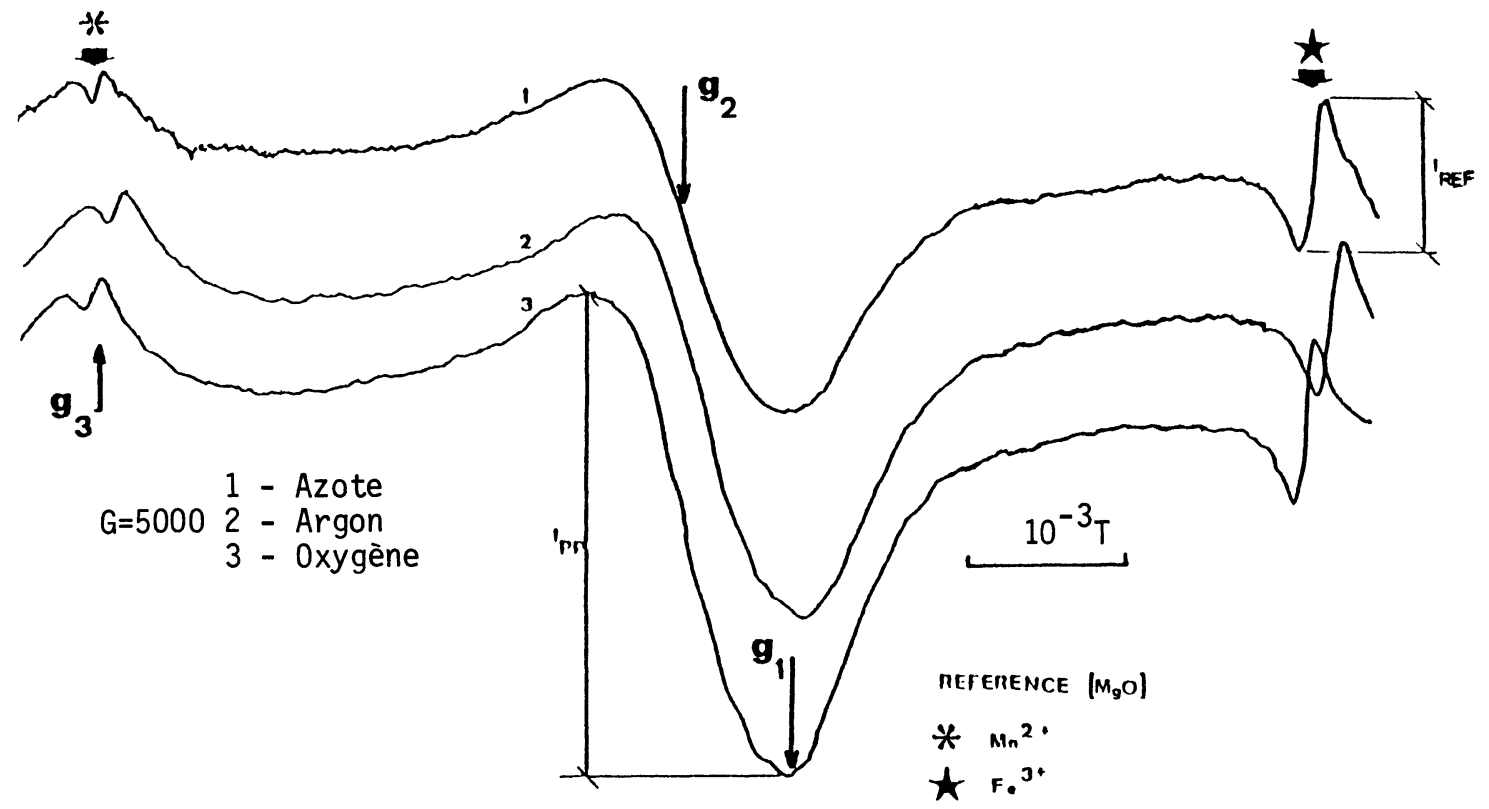

Fig. 4. - ESR spectrum of polypropylene after irradiation by ions $\left(-1-\mathrm{N}_{2}, 2-\mathrm{Ar}, 3-\mathrm{O}_{2}-W_{\mathrm{i}}=40 \mathrm{eV}\right)$.

l'argon et de l'azote. La durée du bombardement a été de $3 \mathrm{~min}$ pour les deux premiers gaz et de 1,5 min pour le troisième, l'énergie $W_{\mathrm{i}}$ étant la même dans les trois cas à savoir $40 \mathrm{eV}$. Les spectres obtenus sont identiques et caractéristiques d'un tenseur $g$ à 3 composantes (Tab. I). Il est à noter que la quantité de radicaux libres crés est plus importante lorsque l'ion provient d'un gaz réactif comme l'oxygène et que l'ion argon plus lourd que l'ion azote, génère plus de dommage.

Lorsqu'on fait croître le temps d'irradiation, l'augmentation de la concentration en radicaux libres semble, comme dans l'évolution des pertes diélectriques, suivre deux cinétiques une saturation très nette étant révélée à partir de $4 \mathrm{~min}$. Cette évolution est quelque peu différente dans le cas de l'oxygène.

Après un bombardement par les électrons la résonance paramagnétique électronique a révélé une faible efficacité de ces espèces par rapport aux ions et ce à surface bombardée égale. La figure 5 permet de comparer les différentes irradiations et en particulier le résultat d'une action simultanée d'un faisceau d'ions de $40 \mathrm{eV}$ et d'électrons de $200 \mathrm{eV}$. Comme nous l'avons indiqué précédemment le spectre enregistré est caractéristique d'un tenseur à trois composantes dont les valeurs calculées sont comparées, dans la table ci-dessous, à celles données par Shimada [5] pour un polypropylène irradié par des rayons $\gamma$. Si l'on prend en compte des travaux antérieurs réalisés avec le polypropylène ayant subi d'autres types de traitement $[6,7]$, il semble que l'on puisse conclure que le radical observé est du type peroxyde. Relatif à un groupement $\mathrm{COO}$ situé dans le plan perpendiculaire à la chaîne du polymère, il

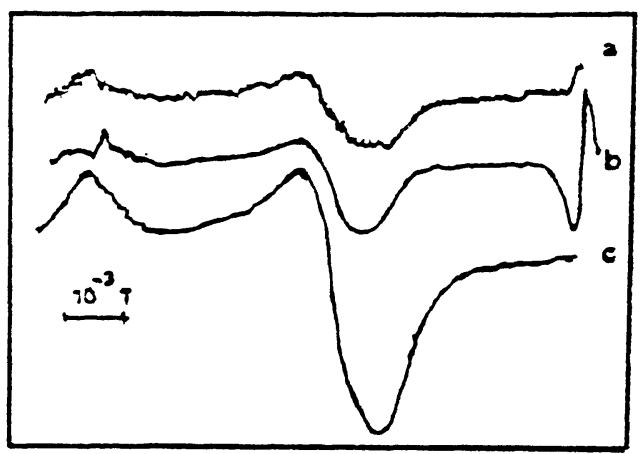

Fig. 5. - ESR spectrum of polypropylene after irradiation. a) by electrons $W_{\mathrm{i}}=250 \mathrm{eV} t=5 \mathrm{mn}$; b) by a glow discharge $t=15 \mathrm{~s} ; \mathrm{c})$ by electrons $(50 \mathrm{eV})$ and ions $(243 \mathrm{eV}) t=5 \mathrm{mn}$.

Table I.

\begin{tabular}{|c|c|c|c|}
\hline $\begin{array}{c}\text { Température } \\
\text { d'observation (K) }\end{array}$ & $g_{3}$ & $g_{2}$ & $g_{1}$ \\
\hline 100 & 2,0027 & 2,0077 & 2,0346 \\
\hline 77 (SHIMADA) & 2,0021 & 2,0081 & 2,0353 \\
\hline
\end{tabular}

peut osciller de manière relativement libre quand il est localisé d'un côté de la chaîne principale du polymère.

3.3 Mesures DE CONDUCTIVITÉ. - Le polymère en feuille irradié dans les mêmes conditions que celles décrites précédemment est placé entre deux 
électrodes planes en acier inoxydable, une lame mince de liquide conducteur est créée entre l'électrode supérieure et la face bombardée du matériau. Cette technique ne permet pas pour l'instant, que la mesure des courants soit faite à l'intérieur de l'enceinte. Une tension continue de polarisation

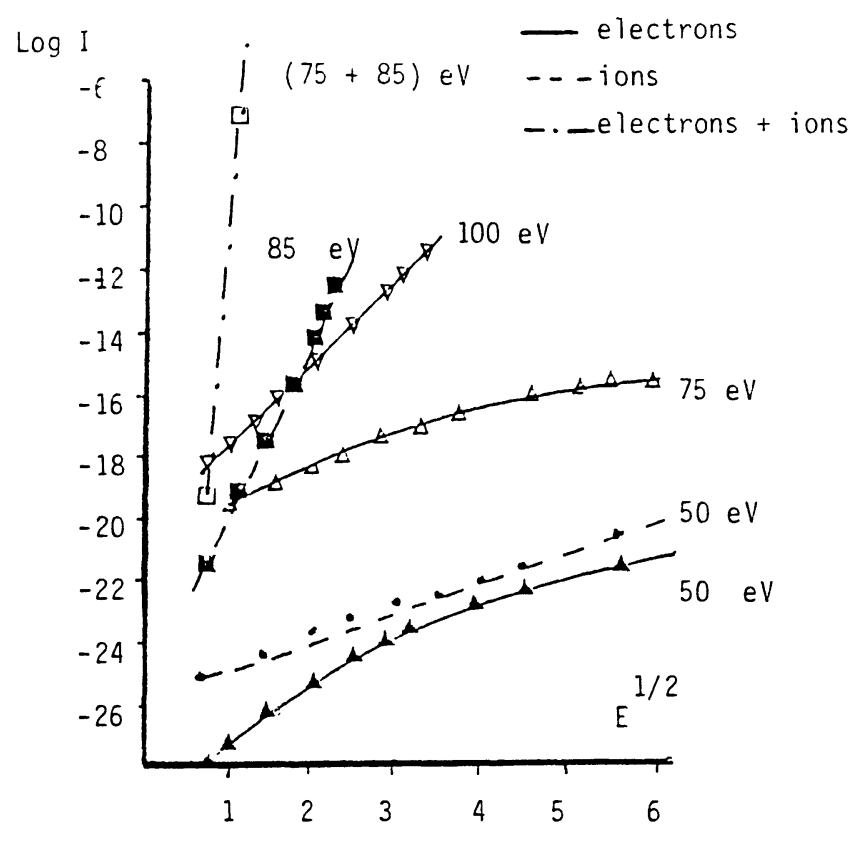

Fig. 6. variable entre 0 et $35 \mathrm{~V}$ a été utilisée. Les courants de fuite mesurés dépendent de cette tension de polarisation, leur amplitude comme le montre la figure 6 est fonction de l'énergie des particules incidentes. On peut noter l'efficacité des ions et des électrons lorsqu'ils agissent simultanément comme ils le font d'ailleurs dans des décharges du type luminescent pour des espaces interélectrodes faibles. Le courant de fuite est de la forme $I=K e^{B E ~ 1 / 2}, K$ et $B$ étant des constantes que l'on définit expérimentalement, $E$ représente le champ électrique.

\section{Conclusion.}

A la faveur de mesures électriques pratiquées à l'intérieur de l'enceinte d'irradiation, nous avons pu constater sur du polypropylène en films le rôle joué par des ions et des électrons de faible énergie et surtout l'efficacité des deux espèces lorsqu'elles agissent simultanément. Une analyse par résonance paramagnétique électronique permet de mettre en évidence la présence de radicaux justifiant en partie les pertes diélectriques mesurées.

Le travail en cours prévoit l'étude du comportement des polymères sous l'action des mêmes espèces et des rayonnements ultra-violet puis des espèces neutres actives. Le dispositif expérimental permettant la production de décharges électriques de divers types, on comparera le comportement des matériaux après le traitement par décharges et après l'irradiation par les espèces ou leur combinaison.

\section{Bibliographie}

[1] Mayoux C., I.E.E.E. Trans. Elec. Insul. EI-11 (1976) 139.

[2] Robinson D. M., Dielectric Phenomena in high Voltage cables, Eds. Chapman and Hall (1936).

[3] Gomès de lima P., Despax B., Mayoux C., BaCQUET G. et Frandon G., Proc. 5th Intern. Symp. Electrets Heidelberg (1985) 494.
[4] Mayoux C., I.E.E.E. Trans. Elec. Insul. EI-12 (1977) 153.

[5] Shimada S., Kotake A., Hori Y. H., KashiwaBARA, Macromolecular 17 (1984).

[6] Fischer H., Hetlwege K. H. et Naudorfl P., $J$. Polym. Sci. Part A. 1 (1963).

[7] Onishi S., Sugimoto S. et NitTa I., J. Polym. Sci. Part A. 1 (1963) 605. 